\title{
Evolução laboratorial da colestase multifatorial em pacientes pediátricos
}

\author{
Laboratorial evolution of multifactorial cholestasis in pediatric patients
}

\author{
Lígia Patrícia de Carvalho Batista Éboli', Marianne Fernanda de Melo Duarte ${ }^{2}$, \\ Luciana Maria Marques de Albuquerque ${ }^{2}$, Thaysa Amaral França ${ }^{2}$, \\ Raquel Nogueira Cordeiro², Diego Laurentino Lima ${ }^{3}$
}

Éboli LPCB, Duarte MFM, Albuquerque LMM, França TA, Cordeiro RN, Lima DL. Evolução laboratorial da colestase multifatorial em pacientes pediátricos / Laboratorial evolution of multifactorial cholestasis in pediatric patients. Rev Med (São Paulo). 2019 set.out.;98(5):309-14.

RESUMO: Introdução: No fígado imaturo do recém-nascido (RN) a colestase neonatal pode ocorrer em associação a um ou vários fatores de risco, sem patologia subjacente. Esta condição denomina-se colestase neonatal multifatorial transitória. Prematuridade, restrição de crescimento intra-uterino ou situações que levam a maior estresse oxidativo (isquemia, comprometimento hemodinâmico, infecções, intervenções cirúrgicas, pausa alimentar, alimentação parenteral e fármacos) estão relacionadas com sua gênese. Objetivo: Avaliar a evolução da colestase neonatal multifatorial quanto a sua duração, assim como verificar as alterações médias das enzimas hepáticas, aspartato aminotransferase (AST), alanina aminotransferase (ALT), e canaliculares, gamaglutamiltransferase (GGT), e da bilirrubina direta (BD), comparando ainda a duração da colestase nos pacientes que fizeram uso do ácido ursodesoxicólico (UDCA) com o tempo de evolução dos que não o fizeram, no intuito de caracterizar melhor essa condição clínica e de chamar a atenção do profissional de saúde sobre sua natureza transitória, evitando dessa maneira a solicitação de exames mais específicos, onerosos e desnecessários. Método: Estudo transversal, com 32 pacientes de zero a 90 dias que apresentavam colestase neonatal multifatorial, acompanhados no ambulatório de hepatologia pediátrica do IMIP, no período de agosto de 2016 a agosto de 2017. Os dados foram coletados através de consulta ao prontuário. A análise estatística dos dados foi realizada por meio do software "R" v.3.3.1. Resultados: A maioria dos pacientes era do sexo masculino $(59,38 \%)$. A idade média de início da colestase foi de 35,5 dias, e a de término de 171,3 dias. A média de duração da colestase foi de 135,8 dias. O valor médio da BD do início e término da colestase foi de 6,05 e $0,29 \mathrm{mg} / \mathrm{dL}$, respectivamente. Com relação às enzimas hepáticas e canaliculares, as médias dos valores de AST, ALT e GGT do início foram 194,7, 123,8 e 387,9 U/L, e de término 69,1, 56 e 121,6 U/L, respectivamente. Apenas 11 pacientes fizeram uso do UDCA. O tempo médio de duração da colestase neste grupo foi de 114,1 dias. Aqueles que não usaram o UDCA, 21 pacientes, apresentaram icterícia colestática por um tempo médio de 147,1 dias. Conclusão: Diante dos resultados obtidos com a análise retrospectiva dos pacientes com diagnóstico de colestase multifatorial, chama a atenção a natureza transitória e benigna desta condição clínica, com duração média da colestase em torno de 136 dias de vida e declínio importante dos níveis das enzimas hepáticas e canaliculares após resolução do quadro.

Descritores: Colestase/diagnóstico; Icterícia neonatal; Recémnascido.

ABSTRACT: Introduction: In the immature liver of the newborn (RN) neonatal cholestasis can occurs in association with several risk factors, with no underlying pathology. This condition is called transient multifactorial neonatal cholestasis. Prematurity, intrauterine growth restriction or situations that lead to increased oxidative stress (ischemia, hemodynamic compromise, infections, surgical interventions, food pause, intravenous nutrition and drugs) are related to its genesis. Objective: to assess the evolution

\footnotetext{
1 Hepatologista pediatra - Instituto de Medicina Integral Prof. Fernando Figueira (IMIP) e da Unidade de Transplante de Fígado do Hospital Universitário Oswaldo Cruz. Gastroenterologista e hepatologista pediatra da Clínica Real Hepato do Real Hospital Português; Tutora do curso de medicina da Faculdade Pernambucana de Saúde (FPS). Éboli LPCB - https://orcid.org/0000-0001-6821-6658. Email: lipcbs@gmail.com.

2 Acadêmica de Medicina da Faculdade Pernambucana de Saúde (FPS), Recife, PE. ORCID: Duarte MFM - https://orcid.org/0000-0002-2261-8763; Albuquerque LMM - https://orcid.org/0000-0003-3750-2891; França TA - https://orcid.org/0000-0003-2161-2850; Cordeiro RN - https://orcid.org/00000002-0238-8374. Email: marianne.fmd@gmail.com, thaysa_amaral@hotmail.com,lu_maria83@yahoo.com.br, raquelnogueiracordeiro@gmail.com

3 Cirurgião Geral. Pesquisador. Professor da disciplina de Prática Hospitalar da Universidade Católica de Pernambuco. Mestrando de Ciências da Saúde da Universidade de Pernambuco, Recife, Brasil. ORCID: https:/orcid.org/0000-0001-7383-1284. Email: dilaurentino@gmail.com

Endereço para correspondência: Raquel Nogueira Cordeiro. Rua dos Coelhos, 300 - Boa Vista. Recife, PE. CEP: 52.050-300. Email: raquelnogueiracordeiro@gmail.com.
} 
Éboli LPCB, et al. Evolução laboratorial da colestase multifatorial em pacientes pediátricos.

of neonatal cholestasis in the multifactorial period, as well as to verify the mean changes in liver enzymes such as aspartate aminotransferase (AST), alanine aminotransferase (ALT), and canalicular, gammaglutamyltransferase (GGT) and direct bilirubin (BD), comparing the duration of cholestasis in patients who used ursodeoxycholic acid with the time of evolution of those who did not, in order to better characterize this clinical condition and to draw the attention of the health professional about its transient nature, thus avoiding the request for more specific, expensive, and unnecessary exams. Methods: A cross-sectional study with 32 patients from zero to 90 days who presented with neonatal multifactorial cholestasis, accompanied in the ambulatory of pediatric hepatology of IMIP, from August 2016 to August 2017. Data were collected through medical records. The analysis of the data was performed using software "R" v.3.3.1. Results: The majority of the patients were male $(59.38 \%)$. The mean age of onset of cholestasis was 35.5 days, and the mean age of onset

\section{INTRODUÇÃO}

colestase neonatal resulta de uma alteração no fluxo biliar canalicular, levando ao acúmulo de substâncias que normalmente são excretadas pelas vias biliares (bilirrubina, ácidos biliares e colesterol) ${ }^{1}$. Caracteriza-se por bilirrubina direta (BD) superior a $1 \mathrm{mg} /$ dl quando a concentração de bilirrubina total for inferior a $5 \mathrm{mg} / \mathrm{dl}$ ou mais do que $20 \%$ do total, se a bilirrubina total for superior a $5 \mathrm{mg} / \mathrm{dl}^{2}$. O termo neonatal é frequentemente usado para se referir à doença hepática colestática que está presente ao nascimento e/ou se desenvolve nos primeiros meses de vida ${ }^{3}$.

No mundo, a frequência da colestase neonatal situase por volta de 1:2.500 nascidos vivos. Existem múltiplas causas de colestase neonatal, muitas vezes relacionadas à resposta do recém-nascido $(\mathrm{RN})$ a agentes endógenos ou condições patológicas específicas ${ }^{4}$. As mais comuns são atresia de vias biliares $(25 \%-40 \%)$, distúrbios genéticos $(25 \%)$ e causas desconhecidas ou multifatoriais ${ }^{5}$.

No fígado imaturo do RN a colestase neonatal pode ocorrer em associação a um ou vários fatores de risco, sem patologia subjacente. Esta condição de início precoce, autolimitada e transitória denomina-se colestase neonatal multifatorial transitória. Prematuridade, restrição de crescimento intra-uterino ou situações que levam a maior estresse oxidativo (isquemia, comprometimento hemodinâmico, infecções, intervenções cirúrgicas, pausa alimentar, alimentação parenteral e fármacos) estão relacionadas com sua gênese ${ }^{6}$.

A investigação laboratorial do RN colestático deve basear-se na anamnese do binômio mãe-criança e obedecer a uma certa hierarquia: as causas mais comuns de colestase e aquelas para as quais existe terapêutica devem ser analisadas inicialmente (descartar atresia de vias biliares é mandatório em todo lactente com icterícia colestática e acolia fecal, uma vez que o diagnóstico precoce desta condição influencia diretamente no seu prognóstico) ${ }^{7}$. Os níveis de enzimas hepáticas, incluindo Alanina was 171 days. The mean duration of cholestasis was 135.8 days. The mean BD value of the onset and end of cholestasis was 6.05 and $0.29 \mathrm{mg} / \mathrm{dL}$, respectively. In relation to liver and canalicular enzymes, the mean values of AST, ALT and GGT at baseline were $194.7,123.8$ and $387.9 \mathrm{U} / \mathrm{L}$, and the mean values of $69.1,56.0$ and $121.6 \mathrm{U} / \mathrm{L}$, respectively. Only 11 patients used ursodeoxycholic acid (UDCA). The mean duration of cholestasis in this group was 114.1 days. Those who did not use the UDCA, 21 patients, presented cholestatic jaundice for a mean time of 147.1 days. Conclusion: In view of the results obtained with the retrospective analysis of patients with a diagnosis of multifactorial cholestasis, the transient and benign nature of this clinical condition is noted, with a mean duration of cholestasis around 136 days of life and with an important decrease in liver enzyme levels after resolution of the condition.

Keywords: Cholestasis/diagnosis; Jaundice, neonatal; Infant, newborn.

Aminotransferase (ALT), Aspartato Aminotransferase (AST), e canaliculares, Gamaglutamiltranspeptidase (GGT) e Fosfatase Alcalina (FA), geralmente são elevados em um neonato colestático e podem sugerir determinadas etiologias $^{8}$.

A colestase muitas vezes não responde à qualquer terapia médica. Porém, alguns estudos evidenciaram sucesso em crianças com doenças colestáticas e uso de Ácido Ursodesoxicólico (UDCA), o qual atua aumentando a formação biliar e antagonizando o efeito de ácidos biliares hidrofóbicos em membranas biológicas ${ }^{9}$.

É importante suspeitar de colestase multifatorial transitória do $\mathrm{RN}$ diante de pacientes que sofreram agravos infecciosos no período neonatal, com necessidade de antibioticoterapia, jejum prolongado ou Nutrição Parenteral Total (NPT), e em situações associadas à hipoxemia, como parada cardiorrespiratória, evitando dessa maneira a realização de exames prematuros, dolorosos e caros para o sistema único de saúde.

Logo, o objetivo desse estudo foi avaliar a evolução da colestase neonatal multifatorial quanto a sua duração, assim como verificar as alterações médias das enzimas hepáticas (AST, ALT) e canaliculares (GGT) e da BD, comparando ainda a duração da colestase nos pacientes que fizeram uso do UDCA com o tempo de evolução dos que não o fizeram, no intuito de caracterizar melhor essa condição clínica e de chamar a atenção do profissional de saúde sobre sua natureza transitória, evitando dessa maneira a solicitação de exames mais específicos, onerosos e neste caso, desnecessários.

\section{MÉTODOS}

Foi realizado estudo transversal analítico cujos dados foram coletados retrospectivamente dos prontuários de pacientes diagnosticados com Colestase Multifatorial acompanhados no ambulatório de hepatologia pediátrica do Instituto de Medicina Integral Prof. Fernando Figueira (IMIP), localizado na cidade de Recife, Pernambuco - 
Brasil, no período compreendido entre agosto de 2016 a agosto de 2017. O estudo teve aprovação no Comitê de Ética em Pesquisa (parecer 1.551.996) e seguiu os preceitos da Resolução 466. Assinatura de termo de consentimento foi solicitada a todos os pacientes e nenhum se negou a participar do estudo.

Foram avaliados 80 pacientes com colestase multifatorial, com idade de zero a 90 dias de vida. Destes, 48 foram excluídos por não apresentarem os dados devidamente registrados nos prontuários, resultando no total de 32 pacientes. A coleta dos dados foi realizada semanalmente durante o período de 6 meses. As pesquisadoras acompanharam o atendimento dos pacientes juntamente com as profissionais responsáveis e após a consulta, registraram os seguintes dados do prontuário: gênero; idade de início, idade de término e duração da colestase; evolução das enzimas hepáticas (AST, ALT) e canaliculares (GGT) e BD; uso ou não de Ácido Ursodesoxicólico (UDCA) no curso da colestase.

Ao final da coleta, os dados foram compilados em tabela de Excel e foram analisados estatisticamente pelo software "R" versão 3.3.1. Para comparar as variáveis numéricas foi utilizado o teste T de student. Considerou-se resultado estatisticamente significante o valor de $p$ menor que 0,05 .

\section{RESULTADOS}

A amostra foi composta por 32 pacientes, sendo $19(59,38 \%)$ do sexo masculino e $13(40,62 \%)$ do sexo feminino. A idade de início da colestase variou entre 1 a

88 dias de vida com média de 35,5 dias, e a de término, de 45 a 464 dias, com média 171.3 dias (Tabela 1).

Nos sexos masculino e feminino, a duração média da colestase foi de 153,4 e 110 dias, respectivamente (Tabela 1). O valor médio de BD do início e término da colestase foi de 6,05 e $0,29 \mathrm{mg} / \mathrm{dL}$, respectivamente (Tabela 2).

Acerca do perfil laboratorial das enzimas hepáticas e canaliculares, observou-se que as médias dos valores de AST, ALT e GGT do início foram 194,7; 123,8 e 387,9 $\mathrm{U} / \mathrm{L}$, e de término 69,$1 ; 56$ e 121,6 U/L, respectivamente (Tabela 3).

Dos 32 pacientes avaliados, 11 fizeram uso do UDCA. O tempo médio de duração da colestase neste grupo foi de 114,1 dias. Aqueles que não usaram o UDCA, 21 pacientes, apresentaram icterícia colestática por um tempo médio de 147,1 dias $(p=0,38)$ (Tabela 4).

Tabela 1 - Idade média de início e término da colestase e duração média da icterícia por sexo

\begin{tabular}{lccc}
\hline Variáveis & Total & Masculino & Feminino \\
\hline Total & 32 & $19(59,38 \%)$ & $13(40,62 \%)$ \\
\hline $\begin{array}{l}\text { Idade média de } \\
\text { início (dias) }\end{array}$ & 35 & 35,1 & 36,2 \\
\hline $\begin{array}{l}\text { Idade média de } \\
\text { término (dias) }\end{array}$ & 171,3 & 188,5 & 142,2 \\
\hline $\begin{array}{l}\text { Duração média } \\
\text { (dias) }\end{array}$ & 135,8 & 153,4 & 110 \\
\hline
\end{tabular}

Tabela 2 - Evolução da bilirrubina direta levando em consideração início e fim da icterícia colestática

\begin{tabular}{cccccc}
\hline Variáveis & Média & Mínima & Máxima & Desvio padrão & $\boldsymbol{p}$ - valor \\
\hline $\mathrm{BD}^{1}{ }_{\text {inicio }}$ & $6,05 \mathrm{mg} / \mathrm{dl}$ & $1,10 \mathrm{mg} / \mathrm{dl}$ & $14,10 \mathrm{mg} / \mathrm{dl}$ & $3,5 \mathrm{mg} / \mathrm{dl}$ & 0,0000 \\
\hline $\mathrm{BD}_{\text {fim }}$ & $0,29 \mathrm{mg} / \mathrm{dl}$ & $0,05 \mathrm{mg} / \mathrm{dl}$ & $0,84 \mathrm{mg} / \mathrm{dl}$ & $0,24 \mathrm{mg} / \mathrm{dl}$ & \\
\hline
\end{tabular}

${ }^{1}$ BD: Bilirrubina Direta Unidade: $\mathrm{mg} / \mathrm{dl}$; Análise estatística: teste T student

Tabela 3 - Evolução das enzimas hepáticas levando em consideração início e resolução da colestase multifatorial

\begin{tabular}{cccccc}
\hline Variáveis & Média & Mínima & Máxima & Desvio padrão & $p$ - valor \\
\hline $\mathrm{AST}^{1}{ }_{\text {inicio }}$ & $194,7 \mathrm{U} / \mathrm{L}$ & $15 \mathrm{U} / \mathrm{L}$ & $675 \mathrm{U} / \mathrm{L}$ & $168,3 \mathrm{U} / \mathrm{L}$ & 0,0001 \\
$\mathrm{AST}{ }_{\text {fim }}$ & $69,1 \mathrm{U} / \mathrm{L}$ & $27 \mathrm{U} / \mathrm{L}$ & $300 \mathrm{U} / \mathrm{L}$ & $60,5 \mathrm{U} / \mathrm{L}$ & \\
$\mathrm{ALT}^{2}{ }_{\text {inicio }}$ & $123,8 \mathrm{U} / \mathrm{L}$ & $6 \mathrm{U} / \mathrm{L}$ & $655 \mathrm{U} / \mathrm{L}$ & $135,5 \mathrm{U} / \mathrm{L}$ & 0,0035 \\
$\mathrm{ALT}_{\text {fim }}$ & $56 \mathrm{U} / \mathrm{L}$ & $20 \mathrm{U} / \mathrm{L}$ & $308 \mathrm{U} / \mathrm{L}$ & $57,0 \mathrm{U} / \mathrm{L}$ & \\
$\mathrm{GGT}^{3}{ }_{\text {inicio }}$ & $387,9 \mathrm{U} / \mathrm{L}$ & $13 \mathrm{U} / \mathrm{L}$ & $3065 \mathrm{U} / \mathrm{L}$ & $554,3 \mathrm{U} / \mathrm{L}$ & \\
$\mathrm{GGT}_{\text {fim }}$ & $121,6 \mathrm{U} / \mathrm{L}$ & $5 \mathrm{U} / \mathrm{L}$ & $822 \mathrm{U} / \mathrm{L}$ & $184,6 \mathrm{U} / \mathrm{L}$ & 0,0087 \\
\hline
\end{tabular}

${ }^{1}$ AST: Aspartato Aminotransferase; ${ }^{2}$ ALT: Alanina Aminotransferase; ${ }^{3}$ GGT: Gamaglutamiltranspeptidase; Unidade: U/L; Análise estatística: teste T student 
Éboli LPCB, et al. Evolução laboratorial da colestase multifatorial em pacientes pediátricos.

Tabela 4 - Duração da colestase comparando os pacientes que utilizaram UDCA com aqueles que não fizeram uso da medicação

\begin{tabular}{ccccccc}
\hline Variáveis & $\mathbf{N}$ & $\begin{array}{c}\text { Média de } \\
\text { duração }\end{array}$ & Mínima & Máxima & $\begin{array}{c}\text { Desvio } \\
\text { padrão }\end{array}$ & p - valor \\
\hline Uso de UDCA & 11 & 114,1 dias & 23 & 196 & 63,8 & 0,3882 \\
Não uso de UDCA & 21 & 147,1 dias & 6 & 463 & 115,3 & \\
\hline
\end{tabular}

${ }^{1}$ UDCA: Ácido Ursodesoxicólico; Análise estatística: teste T student

\section{DISCUSSÃO}

No presente estudo, foram avaliados 32 pacientes com diagnóstico de colestase multifatorial. Albers et al. ${ }^{10}$, avaliando RN que receberam NPT durante pelo menos 7 dias após cirurgia intestinal, concluíram que o sexo masculino predispõe esse paciente cirúrgico à colestase associada à NPT. Simões et al. ${ }^{11}$ também detectaram que o sexo masculino foi predominante nos casos de colestase multifatorial ${ }^{11,12,13}$.

As principais causas da multifatoriedade não foram avaliadas, uma vez que na maioria dos casos ocorre uma junção delas, ou seja, prematuridade associada a baixo peso, muitas vezes secundários a infecções congênitas, ou até sepse precoce com necessidade de antibioticoterapia, jejum prolongado e NPT. Sendo assim, admitiu-se que todos pacientes eram portadores de pelo menos dois fatores de risco para o desenvolvimento da colestase multifatorial.

A faixa etária dos pacientes variou entre 1 e 88 dias de vida, com idade média de início da colestase de 35,5 dias. Dado semelhante foi encontrado por Chen etal. ${ }^{14}$, nos bebês com baixo peso e com colestase associada à NPT, a icterícia colestática apareceu $37 \pm 2,6$ dias após o nascimento. Já em um estudo feito por Kim et al. ${ }^{15}$ a idade de início da colestase foi de 52,4 dias.

$\mathrm{O}$ valor médio da $\mathrm{BD}$ encontrado no início do quadro foi de $6,05 \mathrm{mg} / \mathrm{dl}$ com valor máximo de $14,1 \mathrm{mg} /$ dl. Liu et al. ${ }^{16}$ evidenciaram valor médio de $\mathrm{BD}$ no início da icterícia colestática de $3,45 \mathrm{mg} / \mathrm{dl}$. No estudo de Kim et al. ${ }^{15}$, o valor máximo de BD foi de $6,8 \mathrm{mg} / \mathrm{dl}$.

No atual estudo, sendo estabelecido como nível normal $\mathrm{BD}<1 \mathrm{mg} / \mathrm{dl}$, a duração média da hiperbilirrubinemia direta foi de aproximadamente 136 dias. Liu et al. ${ }^{16}$, em estudo de coorte prospectivo, avaliaram 74 pacientes com icterícia colestática de etiologia multifatorial, sendo estabelecido como critério diagnóstico BD $>2 \mathrm{mg} / \mathrm{dl}$. A duração média da icterícia foi de aproximadamente 86 dias ${ }^{16}$. O tempo menor de duração da colestase encontrado no estudo de Liu et al. ${ }^{16}$ pode ser decorrente do fato de ter sido considerado $\mathrm{BD}$ alterada $>2 \mathrm{mg} / \mathrm{dL}$. É importante ressaltar que o valor de $\mathrm{BD}$ como critério diagnóstico diverge na literatura, sendo considerado icterícia colestática $\mathrm{BD}>1 ; 1,5$ ou $2 \mathrm{mg} / \mathrm{dl}$, como verificado nos estudos de Lane et al. ${ }^{10}$, Albers et al. ${ }^{17}$, Satrom et al. ${ }^{18}$. O consenso mais recente de colestase elaborado pelas Sociedades Norte Americana e Europeia de Gastroenterologia, Hepatologia e Nutrição Pediátrica (NASPGHAN e ESPGHAN), conceitua colestase como valores de BD maior que $1 \mathrm{mg} /$ $\mathrm{dl}$ independente do valor da bilirrubina total ${ }^{5}$. Logo, este foi o valor levado em consideração para elaboração do nosso estudo.

No estudo de Liu et al. ${ }^{16}$, não foi verificada diferença entre os valores médios do início e fim das enzimas hepáticas e canaliculares após resolução da colestase, sendo os valores de início de AST, ALT e GGT 116; 86 e 231 U/L e de término, 111; 82 e $226 \mathrm{U} / \mathrm{L}$, respectivamente. No nosso estudo, embora tenha ocorrido um declínio significativo na evolução das enzimas hepáticas e canaliculares, verificouse que mesmo após a resolução da icterícia colestática, os marcadores de lesão hepática podem permanecer alterados, justificando assim, a importância do protocolo estabelecido pelo IMIP de acompanhar os pacientes com diagnóstico de colestase multifatorial até pelo menos os dois anos de idade, dando alta apenas quando toda bioquímica hepática estiver normal, evitando-se, dessa maneira, que outras hipóteses diagnósticas passem desapercebidas.

O UDCA é um agente colerético hidrofílico que aumenta o fluxo da bile, reduz a toxicidade das membranas por ácidos biliares hidrofóbicos e, portanto, pode diminuir o grau de colestase em algumas condições ${ }^{4}$. No estudo de Chen et al. ${ }^{14}$ realizado com $30 \mathrm{RN}$ prematuros de baixo peso e com doença hepática relacionada à nutrição parenteral admitidos no Hospital Nacional da Universidade de Taiwan, $12 \mathrm{RN}$ no grupo de tratamento e 18 no grupo controle, os pacientes que receberam terapia com UDCA apresentaram um período significativamente mais curto de colestase do que aqueles no grupo controle (62.8 vs 92.4 dias, $p=$ $0,006)^{14}$. Já em um estudo retrospectivo maior e mais recente de Thibault et al. ${ }^{19}$, a terapia com UDCA não foi associada à duração da doença hepática associada à nutrição parenteral. De 118 lactentes elegíveis, 64 receberam UDCA; nestes a colestase durou mais tempo (79 vs. 50 dias, $p=0,001$ ). No entanto, o grupo de tratamento foi significativamente menor e englobavam prematuros extremos, o que é uma grande limitação do estudo ${ }^{19}$. Portanto, embora não haja dados na literatura que comprovem o real benefício do uso de UDCA, a medicação é comumente utilizada na prática clínica. Em nosso estudo, o encurtamento no tempo da duração da colestase naqueles pacientes que fizeram uso de UDCA (147.1 vs 114.1 dias) não foi estatisticamente significante $(p=0.38)$. A impossibilidade de avaliar o tempo de uso da medicação e a dose utilizada por não conter esses 
dados registrados em prontuário, pode ser considerada uma limitação do presente estudo.

Ao procurar artigos na literatura nacional e internacional, a maioria das bases de dados não reconhecem o termo colestase multifatorial como Descritores em Ciências da Saúde (DeCS). Uma grande parte dos artigos aborda de forma isolada a relação da condição clínica com NPT como objetivo principal e cita as demais causas de multifatoriedade quando associadas. Além disso, não foram encontrados muitos estudos que avaliassem a evolução dos parâmetros laboratoriais, o que dificultou a comparação dos resultados obtidos. Ademais, quando encontrado estudos que abordam de forma mais detalhada a condição multifatorial, os mesmos não são atualizados, impossibilitando uma avaliação do cenário atual.

Por ser um estudo retrospectivo, realizado através da análise de prontuários físicos de pacientes diagnosticados com uma condição clínica que ocorre em idade precoce, houve uma dificuldade na coleta das informações dos pacientes, uma vez que muitos pacientes não nasceram ou não tiveram seu diagnóstico inicial no IMIP. Isso resultou em um menor número de variáveis analisadas, além de uma grande quantidade de pacientes excluídos, já que a maioria não tinha registrado os valores dos exames laboratoriais no período de início e resolução da icterícia colestática. Dessa forma, ressaltamos a importância do preenchimento adequado do prontuário.

Tendo em vista a importância do diagnóstico precoce e segmento adequado do paciente com colestase neonatal, caracterizar aqueles que apresentam história clínica e evolução dos níveis das enzimas hepáticas e canaliculares compatível com o quadro de colestase multifatorial torna- se importante, uma vez que pode-se evitar a solicitação de exames mais específicos e dispendiosos que oneram o sistema único de saúde desnecessariamente e espoliam o paciente.

\section{CONCLUSÃo}

Diante dos resultados obtidos com a análise retrospectiva dos pacientes com diagnóstico de colestase multifatorial, chama a atenção a natureza transitória e benigna desta condição clínica, com duração média da colestase em torno de 136 dias de vida e declínio importante dos níveis das enzimas hepáticas e canaliculares após resolução do quadro.

Logo, pode-se sugerir que pacientes com colestase que persiste até 136 dias de vida e que evoluem com queda das enzimas hepáticas, permanecendo assintomáticos ao longo do acompanhamento, não indicando patologias mais graves (como atresia de vias biliares ou doenças metabólicas), principalmente se estiver associado a uma história de prematuridade ou estresse oxidativo no período neonatal, pressupõe etiologia multifatorial, não havendo necessidade de investigação mais aprofundada.

Aqueles que fazem uso do UDCA apesar de encontrar uma diminuição na duração da colestase, o uso do ácido nao se mostrou estatisticamente significante. Porém, faz-se necessária a realização de estudos prospectivos tipo caso controle quanto ao uso da medicação para avaliar sua real eficácia na colestase multifatorial, além de estudos com uma amostra de pacientes maior para melhor caracterização dessa condição clínica.

Grau de participação: Informamos para devido fins que o artigo foi confeccionado em conjunto pelo grupo de autores com o grau de participação seguinte: Coleta de dados (Luciana Maria Marques de Albuquerque, Thaysa Amaral França e Marianne Fernanda de Melo Duarte). Orientação organizacional e sobre a essência, argumentação e relevância do trabalho (Lígia Éboli). Análise, pesquisa dos artigos, leitura e exclusão de pesquisas não pertinentes ao envolvimento do tema escolhido (Ligia Éboli, Marianne Fernanda de Melo Duarte, Luciana Maria Marques de Albuquerque, Thaysa Amaral França). Leitura e escrita do conteúdo (Marianne Fernanda de Melo Duarte, Lígia Éboli, Raquel Nogueira Cordeiro e Diego Laurentino Lima). Revisão do texto quanto a integridade e veracidade quanto as fontes utilizadas (Lígia Éboli, Raquel Nogueira Cordeiro e Diego Laurentino Lima). Dessa forma, o grupo de autores certifica participação conjunta na confecção do artigo, esperando contribuir no tema em questão.

\section{REFERÊNCIAS}

1. Tufano M, Nicastro E, Giliberti P, Vegnente A, Raimondi F, Iorio R. Cholestasis in neonatal intensive care unit: Incidence, aetiology and management. Acta Paediatr. 2009;98(11):175661. doi: $10.1111 /$ j.1651-2227.2009.01464.x.

2. Silva ES, Pó I, Gonçalves I. Colestase neonatal - protocolo de abordagem diagnóstica. Acta Pediatr Port. 2010;41(3):141-3.

3. Loomes KM, Erlichman J. Approach to evaluation of cholestasis in neonates and young infants. UpToDate. 2017 Jun [cited 2017 July 27]. Available from: https://www.uptodate.com/contents/ approach-to-evaluation-of-cholestasis-in-neonates-and-younginfants?source $=$ search_result\&search=Definition $\% 20$ of $\% 20$ multifactorial $\% 20$ cholestasis\&selectedTitle $=1 \sim 150$

4. Best C, Gourley GR. Management of neonatal cholestasis. Therapy. 2009;6(1):75-81. https://doi. org/10.2217/14750708.6.1.75.

5. Fawaz R, Baumann U, Ekong U, Fischler B, Hadzic N, Karpen $S$, et al. Guideline for the evaluation of cholestatic jaundice in infants: joint recommendations of the North American Society for Pediatric Gastroenterology, Hepatology, and Nutrition and the European Society for Pediatric Gastroenterology, Hepatology, and Nutrition. J Pediatr Gastroenterol Nutr. 2017;64(1):154-68. doi: 10.1097/MPG.0000000000001334.

6. Carreira MSP. Fisiopatologia e fatores de risco associados ao desenvolvimento de colestase no período neonatal [dissertação]. Porto: Instituto de Ciências Biomédicas Abel Salazar - Universidade do Porto; 2014. Disponível em: https://pdfs.semanticscholar.org/0d33/ e4df3e43154aa99f2280132187a47b7a47b5.pdf. 
7. Pinto R, Silveira TR. Colestase neonatal: uma abordagem prática. Bol Cient Pediatr. 2016;05(3):92-102. Disponível em: http://www.sprs.com.br/sprs2013/bancoimg/17011817 4030bcped_05_03_a05.pdf.

8. Carvalho E, Ivantes CAP, Bezerra JA. Atresia das vias biliares extra-hepáticas: conhecimentos atuais e perspectivas futuras. J Pediatr. 2007;83(2):105-20. https://dx.doi.org/10.2223/ JPED.1607.

9. Nazer H. Cholestasis Treatment \& Management. 2017 Aug [cited 2017 Oct 27]. In: Medscape. Available from: https:// emedicine.medscape.com/article/927624-treatment

10. Albers M, de Gast-Bakker D, van Dam N, Madern G, Tibboel D. Male sex predisposes the newborn surgical patient to parenteral nutrition-associated cholestasis and to sepsis. Arch Surg. 2002;137(7):789-93. doi: 10.1001/archsurg.137.7.789.

11. Simões AP, Santos MC, Nunes A, Martins M, Bispo MA, Palminha JM. Colestase neonatal: contribuição da clínica e dos exames complementares para o diagnóstico diferencial (dez anos de experiência). Acta Pediatr Port. 1999;30(6):45966.

12. Oswari H, Widjaja R, Rohsiswatmo R, Cleghorn G. Prognostic value of biochemical liver parameters in neonatal sepsis-associated cholestasis. J Paediatr Child Health. 2013;49(1):E6-11. doi: 10.1111/jpc.12053.

13. Repa A, Lochmann R, Unterasinger L, Weber M, Berger A, Haiden N. Aggressive nutrition in extremely low birth weight infants: impact on parenteral nutrition associated cholestasis and growth. PeerJ. 2016;4:e2483. doi: 10.7717/peerj.2483.
14. Chen C, Tsao P, Chen H, Chou H, Hsieh W, Chang M. Ursodeoxycholic acid (UDCA) therapy in very-lowbirth-weight infants with parenteral nutrition-associated cholestasis. J Pediatr. 2004;145(3):317-21. doi: 10.1016/j. jpeds.2004.05.038.

15. Kim A, Lim R, Han Y, Park K, Byun S. Parenteral nutritionassociated cholestasis in very low birth weight infants: a single center experience. Pediatr Gastroenterol Hepatol Nutr. 2016;19(1):61-70. doi: 10.5223/pghn.2016.19.1.61.

16. Liu P, Guo L, Huang L, Zhao D, Zhen R, Yuan X, et al. Analysis of factors affecting the prognosis of neonatal cholestasis. Int J Clin Exp Med. 2015; 8(5): 8005-9. Available from: https://www.ncbi.nlm.nih.gov/pmc/articles/ PMC4509307/.

17. Lane E, Murray K. Neonatal cholestasis. Pediatr Clin North Am. 2017;64(3):621-39. doi: 10.1016/j.pcl.2017.01.006.

18. Satrom K, Gourley G. Cholestasis in preterm infants. Clin Perinatol. 2016;43(2):355-73. doi: 10.1016/j.clp.2016.01.012.

19. Thibault M, McMahon J, Faubert G, Charbonneau J, Malo J, Mohamed I, et al. Parenteral nutrition-associated liver disease: a retrospective study of ursodeoxycholic acid use in neonates. J Pediatr Pharmacol Ther. 2014;19(1):42-8. doi: 10.5863/1551-6776-19.1.42.

Recebido: 23.07 .18

Aceito: 28.08 .19 